\title{
Impurity: pharma market and importance
}

\section{Mini review}

The control of pharmaceutical impurities is currently a critical and challenging issue to the pharmaceutical industry. Impurities in pharmaceuticals are the unwanted chemicals that remain with the active pharmaceutical ingredients (APIs), or develop during formulation, or upon aging of both API and formulated APIs to medicines. ${ }^{1}$ The presence of these unwanted chemicals or impurities can have unwanted pharmacological or toxicological effects even in small amounts may influence the efficacy and safety of the pharmaceutical products. ${ }^{2}$

The International Conference on Harmonization (ICH) has formulated a workable guideline regarding the control of impurities. According to $\mathrm{ICH}$, an impurity in a drug substance is defined as-"any component of the new drug substance that is not the chemical entity defined as the new drug substance". ${ }^{1}$ There is an ever increasing interest in impurities present in APIs recently, not only purity profile but also impurity profile has become essential as per various regulatory requirements. ${ }^{3}$

Impurity profile describes the identified and unidentified impurities present in a new drug substance. Impurity profiling is the common name of a group of analytical activities, the aim of which is the detection, identification/structure elucidation and quantitative determination of organic and inorganic impurities as well as residual solvents in bulk drugs and pharmaceutical formulations. ${ }^{2}$ It helps identifying the impurity present in pharmaceutical formulation by analytical technique or methods. Numbers of impurities present in formulation such by-products, degradation products, interaction products, intermediates, penultimate intermediates, related products, transformation product. ${ }^{2,4}$

According to USP impurities have various sections which are Impurities in official Articles, ordinary impurities, and organic volatile impurities. ${ }^{5}$ According to ICH impurities occurred or produced by chemical syntheses which are organic impurities (Process and Drug related), Inorganic Impurities, and Residual Solvents. ${ }^{1}$ There are various sources of impurity like heavy metals, ligands, catalysts other materials like degraded end products obtained during or after manufacturing of bulk drug or products. ${ }^{2,4,6}$ As looking to the scenario of API and Pharma market it is also important to have the impurities for safety and efficacy of drug as per the stringent guideline of regulatory authorities. Impurity synthesis is upcoming market in next few years and which will play a vital role in Pharma industry. ${ }^{1}$ Impurity synthesis is nothing but to synthesize the molecules which were unpredicted or byproduct which are very tough to synthesize. Key player in impurity synthesis market includes Toronto Research Chemicals, Molcan, TLC Pharmaceutical Standards, Veeprho Laboratories Pvt. Ltd., Clearsynth, Opulent Pharma, CleanChem Laboratories etc. ${ }^{3,7}$

In impurity profiling identification plays crucial role and Methods for impurity detection are Isolation and characterization, Column chromatography, Gas chromatography, Flash chromatography, TLC, GC, HPLC, HPTLC, Capillary electrophoresis (CE). ${ }^{6-8}$

Following Characterization of impurity also plays important role in impurity profiling:
Volume I Issue 4 - 2017

\author{
Amol Kulkarni, 'Vrushali A Kulkarni² \\ 'CAYMET's Siddhant College of Pharmacy, India \\ ${ }^{2}$ Modern College of Pharmacy, India
}

Correspondence: Amol A Kulkarni, M Pharm, Ph.D, Director Research, CAYMET's Siddhant College of Pharmacy, Sudumbare, Pune-4I2109, India, Email dramolk301@gmail.com

Received: June 24, 2017| Published: September 05, 2017

\author{
i. NMR \\ ii. Mass spectroscopy \\ iii. LC-MS \\ iv. GC-MS
}

i. Nuclear magnetic resonance (NMR): Detection of impurity is also challenging while looking at complex or multicomponent mixture analysis of API. ${ }^{2}$ NMR plays an important role in structural detection of impurities which provides information about specific bonding between peak area and number of nuclei responsible for peak. NMR spectroscopy is used to identification and structure elucidation the structure of impurity present in API. ${ }^{8}$

ii. Mass spectroscopy (MS): Mass spectroscopy is a most accurate method for determining the molecular mass of the compound and its elemental composition of impurities. Mass spectroscopy is used to prove identity of structure, give exact molecular mass, give molecular formula and most important for structure elucidation of new compound. ${ }^{2}$ Now a day's mass spectroscopy connected with various hyphenated techniques like GC-MS, LC-MS, LCMS-MS HPLC-DAD-MS, HPLCDAD-NMR-MS, Tandem Mass spectroscopy and capillary electrophoresis-Mass spectroscopy and these techniques deliver very high sensitivity up to picogram level with high separation efficiency. ${ }^{6-8}$

iii. GC-MS: To identify different substances within a test sample gas chromatography-mass spectrometry (GC-MS) method used, that combines the features of gas-liquid chromatography and mass spectroscopy. In this method gas chromatography separate volatile and semi-volatile compounds with great resolution. Mass spectrometer can provide detailed structural information on most compounds such that they can be exactly identified, but it cannot readily separate them. Sample vaporized by injection into a heated system, eluted through a column by inert gaseous mobile phase and detected. The sample is transported through the column by the flow of an inert, gaseous mobile phase, the carrier gas. Flow is regulated by the pressure regulators and gas metering valves. GC operates at atmospheric pressure and the MS ion source at 10-5 Torr.108 fold pressure difference. The carrier gas must be removed and GC peak components transferred to the MS ion source.? 
iv. LC-MS: $\mathrm{LC} / \mathrm{MS}$ is a hyphenated technique, combining the separation power of HPLC/UPLC, with the detection power of mass spectrometry. ${ }^{6} \mathrm{LC} / \mathrm{MS}$ became really popular with the introduction of the thermo spray interface and the particle beam interface. This is same as GC-MS but removal of liquid carrier from an HPLC eluent before samples are passed in to the MS source. To handle normal eluent flow rate $0.5-2.0 \mathrm{ml}$ min $^{-1}$ which is not handled by MS pumping system moving belt inlet systems, jet separators and vacuum nebulizers are used. $^{7}$

According to a New Market Report published by Persistence Market Research "Active Pharmaceutical Ingredient (API) Market" the Global Active Pharmaceutical Ingredient Market was valued at US\$ 143 Bn in 2016 and is Estimated to Reach US\$ 186 Bn by 2020 at a CAGR of $6.6 \%$ From 2015 to $2020 .^{9}$

Increase in global incidences of lifestyle related diseases and development of high potency APIs will continue to positively influence the global API market in 2017. Demand for active pharmaceutical ingredients is also growing due to increase in the geriatric population globally. ${ }^{10}$

Key players in the global API market include Teva Pharmaceutical Industries Ltd., and Zhejiang NHU Co., Ltd, Zhejiang Medicine Co. North China Pharmaceutical Group Corp. (NCPC) and Northeast Pharmaceutical Group Co, Dr. Reddy's Laboratories Ltd., Aurobindo Pharma, Sandoz (Novartis AG), Zhejiang Huahai Pharmaceutical Co.,Ltd, Ltd and Zhejiang Hisun Pharmaceutical Co., Ltd. ${ }^{11-15}$

\section{Conclusion}

This study will help to a researcher/investor who wants to work on impurity profiling. It gives idea about the impurity profiling, different types. This gives brief information about isolation and characterization, different methods of impurity detection and market share of API and future of impurity.

\section{Acknowledgements}

None.

\section{Conflict of interest}

The author declares no conflict of interest.

\section{References}

1. International Conference on Harmonisation. ICH Harmonized Tripartite Guidelines, Impurities in New Drug Substances Q3A(R1). International Conference on Harmonisation of Technical Requirements for Registration of Pharmaceuticals for human use. 2002.

2. Camarasu C, Madichie C, Williams R. Recent progress in the determination of volatile impurities in pharmaceuticals. Trends Anal Chem. 2006;25(8):768-777.

3. Lee H. Pharmaceutical industry practices on genotoxic impurities. USA: CRC Press; 2014.

4. McGovern T, Jacobson KD. Regulation of genotoxic and carcinogenic impurities in drug substances and products. Trends Anal Chem. 2006;25(6):790.

5. United States Pharmacopeia Convention. USA: Rockville; 2006.

6. Gorog S. Identification and Determination of Impurities in Drugs. 1st ed. Amsterdam, Netherlands; 2000. p. 1-772.

7. Akhtar G. Indian pharmaceutical industry: an overview. IOSR J Humanit Soc Sci. 2013;13(3):51-66.

8. Szántay C, Béni Z, Balogh G, et al. The changing role of NMR spectroscopy in off-line impurity identification: a conceptual view. Trends Anal Chem. 2006;25(8):806-820.

9. Global Market Study on Active Pharmaceutical Ingredients: Small Molecules API Segment Anticipated to Create Significant Growth Opportunities During 2017-2025. Life Sciences \& Transformational Health. 2017:256.

10. Snyder RD, Green JW. A review of the genotoxicity of marketed pharmaceuticals. Mutat Res. 2001;488:151-169.

11. Baertschi SW. Analytical methodologies for discovering and profiling degradation-related impurities. Trends Anal Chem. 2006;25(8):758.

12. Abrol D, Prajapati P, Singh N. Globalization of the Indian pharmaceutical industry: implications for innovation. Institutions and Economies. 2011;3(2):327-365.

13. European Pharmacopoeia. 4th ed. Council of Europe. France: Strasbourg; 2004.

14. Olsen BA, Castle BC, Myers DP. Advances in HPLC technology for the determination of drug impurities. Trends Anal Chem. 2006;25:796.

15. Lee MS, Kerns EH. LC/MS applications in drug development. Mass Spectrom Rev. 1999;18(3-4):187-279. 